DOI: https://doi.org/10.47405/mjssh.v6i12.1203

\begin{tabular}{|c|c|}
\hline 4 & Malaysian Journal of Social Sciences and Humanities (MJSSH) \\
\hline 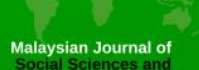 & Volume 6, Issue 12, December 2021 \\
\hline (MJ-sSH) & e-ISSN : 2504-8562 \\
\hline & $\begin{array}{l}\text { Journal home page: } \\
\text { www.msocialsciences.com }\end{array}$ \\
\hline
\end{tabular}

\title{
The Importance of Health Information Seeking Among Diabetes Patients in Malaysia: A Preliminary Observation
}

\author{
Nadia Amirudin"1, Siti Nurhanim Mohamed Aimanan', Rosnani Kassim¹, Norbaidurah Ithnain"1, \\ Albeny Joslyn Panting1 \\ ${ }^{1}$ Institute for Health Behavioural Research, Ministry of Health Malaysia, Malaysia
}

Correspondence: Nadia Amirudin (nadia.amr@moh.gov.my)

\begin{abstract}
The sharing of health information by educating patients and encouraging them to seek relevant information is one of the main strategies in disease prevention. By evaluating patient's information seeking behaviour and identifying the health information seeking trends, it is possible to establish effective techniques to deliver the necessary information to the patients to enhance their selfmanagement skills. It is critical to keep people informed about effective preventative techniques that can control their disease progression. Thus, this study aimed to highlight the role and importance of health information seeking behaviour among people with diabetes in Malaysia.
\end{abstract}

Keywords: Health information seeking behaviour, diabetes, role, theory, health literacy

\section{Introduction}

The prevalence of diabetes is expected to increase globally across all countries in the next 25 years, with the largest increases projected to be from middle-income countries. According to the International Diabetes Federation's (IDF), three out of four people who are living with diabetes (352 million people) are in the productive age group between 20 and 64 years old as reported in $9^{\text {th }}$ edition Diabetes Atlas, 2019. In Southeast Asia, the prevalence of diabetes is expected to rise from $11.3 \%$ in 2019 to $12.2 \%$ in 2030 and further on to $12.6 \%$ in 2045.

Malaysia is one of 39 nations categorised under the IDF Western Pacific region. It is also one of the world's diabetes hotspots with as high as 3.6 million cases. Based on the National Health and Morbidity Survey (NHMS), there is a growing trend of adult diabetes patients above the age of 18 from $11.6 \%$ in $2006,15.2 \%$ in $2011,17.5 \%$ in 2015 to $18.3 \%$ in 2019 . Furthermore, a substantial difference in the diabetes prevalence was detected among Malaysia's major ethnic groups, with Indians having the highest prevalence of Type 2 Diabetes Mellitus (T2DM), followed by Malays and Chinese.

As a chronic condition, patients' perspectives on information and knowledge of diabetes are critical in managing the disease to give rise to diabetes self-management education (DSME) and ongoing support (Kalantzi et al., 2015). Understanding Information Seeking Behaviour (ISB) among diabetes patients is vital because the relevant knowledge enables the patients to engage in more self-care behaviours to manage their diabetes more successfully, subsequently improving their quality of life. An in-depth comprehension of this dynamic process will allow the development of effective interventions for diabetes patients, especially in the effort to enhance their health literacy with regard to self-care diabetes 
management. Thus, it is necessary to analyse the information-seeking behaviour (ISB) of individuals with T2DM to provide practical recommendations in the development of health education programmes that will adequately support people living with diabetes.

\section{Health Information Seeking Behaviour (HISB)}

According to Wilson (2000, p56), ISB refers to "purposive information searching as a result of a desire to do something". During the information search, the individual may engage with manual information systems such as a newspaper or a library, or with computer-based information systems; the World Wide Web. ISB is a dynamic concept used to understand T2DM patients' behaviour in terms of what information they seek, from where they seek it (the source), the specific types of information they prefer, the barriers they face while seeking information, their needs in seeking health information, and the frequency of seeking health information (Lambert et al., 2007).

Health information seeking behaviour (HISB) is gradually garnering public attention throughout the world, including Malaysia (Maon et al., 2017). It is frequently undertaken in response to a health problem or fear about a health problem (Lagoe, \& Atkin, 2015). According to Niederdeppe et al. (2007), HISB refers to the process of obtaining information about health treatments, alternative medicine, diet, and physical activity. On the other hand, HISB might be investigated in various circumstances, including dealing with a health-threatening crisis, participating in medical decision-making, and engaging in preventative behaviour. Furthermore, it is frequently referred to as a problem-focused coping technique, thus suggesting that individuals with HISB devote their efforts towards becoming more dedicated.

Moreover, Zhang et al. (2020) claimed that information seeking might help individuals to improve their coping abilities by enabling them to access relevant, high-quality health information that a guide them in medical decision-making. HISB is also envisioned as a mechanism for generating the type and quantity of information deemed vital for an individual to participate in medical decision-making. Individuals who actively participated in decision-making with healthcare professionals are likely to be more engaged in their search for health-related information (Vahdat et al., 2014). Nonetheless, a study conducted by Hashimoto and Fukhara (2004) discovered that the request for information does not always imply an active role in decision-making; rather, information may be sought for other goals such as evaluating the proposed therapy or forecasting the sequence of events.

Besides that, HISB is also identified as a significant factor that influences a person's willingness to engage in a healthy lifestyle or preventative behaviour. While obtaining appropriate information does not ensure healthy behaviour, it may empower individuals to make beneficial adjustments in health management (Shi et al., 2004). According to Hamzah et al. (2015), the understanding of health information seeking behaviour can offer insight into the present condition of the disease and also identify potential gaps in this area, particularly with regard to demographic characteristics. Similarly, the same study highlighted that research on information seeking behaviour may cast an effect on people's ability to control their health, particularly those with chronic diseases such as diabetes, cancer, or cardiovascular disease.

In Malaysia, health promotion is viewed as a prerequisite in the control of chronic conditions. However, despite the availability of numerous health education initiatives, existing barriers lead to continuous escalation of chronic disease cases. In addition, many of the elements that may obstruct health seeking behaviours are unknown (Yu et al., 2015). To date, relatively little HISB research has been conducted in Malaysia, particularly among people with diabetes (Niederdeppe et al., 2007; Hamzah et al., 2015). Thus, the strengthening of health promotion activities is vital to empower patients' knowledge and capacity with regard to health information seeking (Mathialagan et al., 2018), particularly for diabetes patients in Malaysia.

Investigating the channels of information sources may assist the development of an effective delivery method of health-related information to assist any relevant parties in providing concise content that can 
be easily comprehended by the average person (Longo et al., 2010; Hamzah et al., 2015). Different approaches are required for marketing and information delivery purposes. These efforts must be intensive and tailored to the distinct needs of individuals to engage and motivate them in HISB for improved disease management (Lintonen et al., 2008). To achieve this, research is warranted on various aspects, including language preference to adapt to the needs of a particular population (Ahmad et al., 2014; Kueh \& Kuan 2018), improvised use of various media in broadcasting, professionally designed educational programmes (Qusaier et al., 2017), and exploitation of the internet to share and exchange information (Ahadzadeh et al., 2017).

In the literature, most of the related research explored the patterns of HISB, specifically on how people interact with information, for instance; how they seek and use information. However, relatively few studies on HISB among diabetes patients in Malaysia have been conducted to date. Thus, a study examining trends in HISB, with a particular emphasis on diabetes patients seeking treatment at government health clinics in Malaysia, is crucial for overall improvement to overcome medical-related challenges.

\section{Model of Health Information Seeking Behaviour}

Health information seeking models are used to anticipate the behaviours and behavioural patterns of the people while seeking health-related information. Patients make self-management decisions based on their current needs by acquiring and integrating the relevant knowledge that can assist them to take the actions deemed essential at the time. Wilson's Information-Seeking Behaviour Model (1981, 1996, 1999) provides a detailed conceptual model of HISB. The model is useful in examining the interactions between the variables that influence the HISB, determining whether the patient is an active or passive information seeker, and ascertaining the effects of HISB on health outcomes. Although the model was developed with cancer patients in mind, it can be modified to a certain extent to accommodate individuals with other chronic diseases such as diabetes and cancer (Longo et al., 2010).

According to Wilson's Information-Seeking Behaviour Model, information-seeking behaviour can be categorised into four distinct categories: passive attention, passive search, active search, and ongoing search (Wilson, 1997). Passive attention refers to individuals who obtain knowledge inadvertently from actions such as watching television. Passive searching entails obtaining pertinent information while conducting other types of searches. It can evolve into an active search mode, particularly when the subject is of interest to the individuals and they are eager to learn more. Lastly, an ongoing search occurs during an active search when patients need to update their knowledge, confirm something, or satisfy their curiosity with regard to previously obtained information. Both contextual and personal characteristics of patients affect their HISB that in turn has an effect on both active and passive information seeking, as well as their experiences.

However, according to Lenz's information seeking model, the information search process is viewed as a subset of the decision-making process. The length of time and complexity of the selection process are assumed to be different and revocable at any stage. In general, the information-gathering component of the overall decision-making process consists of six sequential stages. Firstly, a stimulus, derived from within the person or the external environment is considered as the "call to action" that initiates the process of search. Recognising a problem to be solved, making a decision, achieving a goal, or anticipating a dangerous situation are all common stimuli for individuals to search for health-related information. Secondly, goal setting is performed to ensure the search will lead to more focused information to prevent unnecessary activities. Based on these objectives, the patient then determines the time frame for gathering information, the information sources to be used, the types of information needed, and other relevant factors.

Thirdly, the decision-making about active search is determined by the amount of prior knowledge provided, the mental background of the issue, and the expected cost-benefit ratio of information seeking. If people believe that they have obtained sufficient information, they will not strive for more. Fourthly, the search behaviour is the stage that occurs when a person decides to actively seek 
information. The scope of search is determined by two factors, such as the number of alternatives investigated and the number of aspects for each alternative. The extent of the search can range from superficial to detailed information gathering. It is also relative to the quantity of information obtained.

Next, after actively seeking information, the individual will enter the stage of information acquisition. They will evaluate the information gathered and decide whether the current information obtained is meaningful or meaningless. Any current and fresh information is more easily remembered and it will act as a stimulus to encourage further search behaviour. Lastly, a decision will be made based on the adequacy of the new information acquired. The sufficiency of this new information is assessed to determine whether information seeking should be continued or discontinued. The end result of this search process can be seen from the cognitive and behavioural changes of the information seeker.

Besides that, the health information acquisition model of Freimuth, Stein, and Kean also has six stages, similar to Lenz's information seeking model. Lenz (1984) and Freimuth et al. (1989) also provided the most solid understanding of several aspects of HISB, including the extent and method. These steps are part of a decision-making process in which the selection at each phase influences whether one may go to the next level or the previous level must be repeated. However, this model does not study personal or environmental determinants that may affect information seeking behaviour. On the contrary, the strength of this model lies in the refinement of the information-seeking process. If the amount of information acquired is insufficient, one should return to the cost-benefit analysis step and continue the process until a satisfactory outcome is achieved.

Longo (2005) and Johnson (1997), on the other hand, focused heavily on the technique dimension. Johnson explained the technique dimension in detail, emphasising that information sources are chosen based on how well they fulfil an individual's information demands. Most of the models examined focus solely on whether people seek information or not, but neglect the potential of diversity within different extremes of HISB. Longo's (2005) model is the only one that takes into account the varied outcomes of information-seeking process. Collectively, all of the models give valuable insights into HISB, especially improving the understanding of why some people seek accessible knowledge while others do not.

\section{Roles of Information Seeking Behaviour}

Various individuals seek health-related information for different reasons. Today, many people search for health-related information to improve their health and quality of life. Numerous studies have discovered a positive correlation between individuals' health awareness and their HISB. If patients seek information to improve their health, this information will be included in the decision-making process for health-related decisions. Thus, it can be characterised as a health decision-making process. As a consequence, patients feel more empowered and become more involved in their health-related decisionmaking (Jacobs et al., 2017). Ultimately, it may improve treatment effectiveness and patient satisfaction. Besides, inappropriate consultations with healthcare providers can be reduced when patients can monitor their symptoms based on credible and verifiable health information. It may also result in cost savings associated with transportation and communication. In addition, patient's ability to communicate with their doctors and engage in clinical consultations will be enhanced. Hence, patients will establish a new perspective on the doctor-patient relationship (Alwi et al., 2018). Moreover, HISB can be tailored to the unique characteristics of each individual based on their health status and concerns. In addition, patients can regulate their learning rates by seeking health information at their own pace, thus avoiding information overload during consultations with their doctors.

Despite that, direct communication between doctors and healthcare providers is not the only source of information for patients. Some patients may actively seek out health information to gain new insights on how to improve their health situation, before seeking advice from medical practitioners regarding this new information. Additionally, information seeking behaviour is seen as a useful method to respond to health-improvement activities and psychological adaption to the illness. It is also a type of coping mechanism to deal with uncertainty, adapt to change, and reclaim control following a medical incident. 
Furthermore, patients' information-seeking behaviour can be used to ascertain the factors that motivate people to seek pertinent information (Milewski et al., 2010).

Furthermore, the examination of patients' information-seeking behaviour enables the development of strategies for a successful transmission of health information to patients with the aim of significantly increasing support for their self-care. Self-management is a critical component of treatment and patients need to increase their awareness and knowledge of their health condition. Minimising barriers to seeking, locating, and utilising diabetes-related information is critical to reducing the severity of the disease to improve the patient's health status. It can also be used to sway the opinions of medical experts and healthcare professionals so that better mutual understanding can be fostered between patients and physicians and to reduce miscommunication, ultimately reducing other complications.

\section{Information Seeking Behaviour and Health Literacy}

During the Ninth Global Conference on Health Promotion in Shanghai, Malaysia and many other countries endorse the Shanghai Declaration that acknowledges health literacy as an important predictor of health and commits to its development (World Health Organization, 2017). Malaysia has committed to designing, implementing, and monitoring various national initiatives aimed at enhancing health literacy across all demographics and educational settings. Shaw et al. (2009) asserted that health literacy is a cultural construct that is strongly influenced by social factors. By providing the additional meaning of health information based on cultural factors, an individual's viewpoint and definition of health and illness can be modified. As a result, this cast an impact on how people perceive health literacy in various countries and contexts. While the importance of information demands among diabetes patients is undeniable, it remains understudied, particularly when considering the heterogeneity of diabetes communities and diabetes patients at various phases of the disease (Biernatzki et al., 2018). Additionally, low health literacy was associated with an inability to evaluate online health information (Diviani et al., 2015). In other words, health literacy is critical and an essential skill for anyone seeking health information (Ishikawa, 2008).

Additionally, the understanding of information seeking behaviour is crucial to offer perspective and insight in all spheres of life. In Malaysia, the National Health and Morbidity Survey (NHMS) 2019 indicated that $35 \%$ of persons aged 18 years and above showed inadequate health literacy, thus reflecting a poor level of health knowledge among Malaysian adults. Furthermore, individual health literacy is a significant determinant of an individual's ability to obtain health information that can assist them in making educated health decisions (Abdullah et al., 2019). In addition, a few research revealed that the incidence of low health literacy among patients with T2DM in Malaysia to be as high as 85.8 percent (Azreena et al., 2016).

Moreover, a study by Abdullah et al. (2020) reported that only 14.2\% of Malaysian patients with T2DM surveyed in the primary care clinics have adequate health literacy, as compared to $23.7 \%$ in Taiwan. The observed discrepancy could be explained by a multitude of variables. Critical assessments of health education programmes offered to T2DM patients in Taiwan and Malaysia, as well as comparisons of the factors associated with lower levels of health literacy in these two countries, may provide some answers. On the other hand, the determination of the level of health literacy and pattern of information seeking behaviour among T2DM patients may assist policymakers and healthcare providers in identifying the barriers and strategies related to active health seeking behaviours so that improved healthcare information delivery and health education strategies can be implemented to improve patients' quality of life.

\section{Conclusion}

A study of information seeking behaviour is valuable for the improvement of public health, and more importantly, for individuals' ability to self-manage their health. Moreover, the involvement of healthcare providers apart from doctors, such as dieticians and diabetes educators also represent a significant 
opportunity to provide better information for diabetes patients. Therefore, obtaining the relevant pertinent knowledge is requisite in influencing individuals' behaviour to improve their current state of health, particularly individuals who are suffering from chronic diseases such as diabetes. In contemporary lifestyles, self-management is closely related to information seeking behaviour, especially those related to health. Understanding how information seekers with chronic diseases behave can be beneficial in guiding healthcare providers to provide relevant materials or additional information that is succinct, clear, and understandable.

\section{Acknowledgment}

We would like to thank the Director-General of Health and Deputy Director-General of Health (Research and Technical Support), Ministry of Health Malaysia for the permission to publish this paper.

\section{Competing Interests Statement}

The authors declare that there are no competing or potential conflicts of interest.

\section{References}

Abdullah, A., Liew, S. M., Salim, H., Ng, C. J., \& Chinna, K. (2020). Health Literacy Research in Malaysia: A Scoping Review. Sains Malaysiana, 49, 1021-1036. https://doi.org/10.17576/jsm$\underline{\text { 2020-4905-07 }}$

Abdullah, A., Liew, S. M., Salim, H., Ng, C. J., \& Chinna, K. (2019). Prevalence of limited health literacy among patients with type 2 diabetes mellitus: A systematic review. PLOS One, 14(5), e0216402. https://doi.org/10.1371/journal.pone.0216402

Abdulrahim Qusaier, R., Al-Tqiqi, Y. E., Al-Ahmadi, S., Al-Qarni, A. M., \& Felemban, A. M. (2017). Information Seeking Behaviour among Diabetic Patients Attending Primary Healthcare in Western Saudi Arabia: A Cross-Sectional Two-Center Study. Original Research Article, 41(1), 41-50. https://doi.org/10.21276/ijmrp.2017.3.1.008

Ahadzadeh, A. S. (2017). Online Health Information Seeking among Malaysian Women: Technology Acceptance Model Perspective. SEARCH: The Journal of the South East Asia Research Centre for Communications and Humanities, 9(1), 47-70.

Ahmad, B., Ramadas, A., Kia Fatt, Q., \& Zain, A. Z. M. (2014). A pilot study: the development of a culturally tailored Malaysian Diabetes Education Module (MY-DEMO) based on the Health Belief Model. BMC Endocrine Disorders, 14(1). https://doi.org/10.1186/1472-6823-14-31

Alwi, S.S.E. \& Murad, M.A.A (2018). 'Online Information Seeking: A Review of the Literature in the Health Domain'. World Academy of Science, Engineering and Technology, Open Science Index 144, International Journal of Computer and Information Engineering, 12(12), 1025 - 1031.

Azreena E, Suriani I, Juni MH \& Fuziah P. (2016). Factors associated with health literacy among Type 2 Diabetes Mellitus patients attending a government health clinic, 2016. Int J Public Heal Clin Sci. ;3(6): 50-64.

Biernatzki, L., Kuske, S., Genz, J., Ritschel, M., Stephan, A., Bächle, C., \& Icks, A. (2018). Information needs in people with diabetes mellitus: a systematic review. Open Access Bio Med Central (BMC), 7(1), 27https://doi.org/10.1186/s13643-018-0690-0

Diviani, N., van den Putte, B., Giani, S., \& van Weert, J. C. (2015). Low health literacy and evaluation of online health information: A systematic review of the literature. Journal of Medical Internet Research, 17(5), e112. https://doi.org/10.2196/jmir.4018

Freimuth, V.S., Stein, J.A., \& Kean, T.J. (1989). Searching for health information: The cancer information service model. Philadelphia: University of Pennsylvania Press. 
Ganapathy, S. \& Jawahir, S. \& Jailani, A. \& Bakar, S. (2020). National Health and Morbidity Survey 2019: Technical Report - Volume II (Healthcare Demand). https://doi.org/10.13140/RG.2.2.16717.05603

Hamzah, M. R., Mohammad, E.M.W., Abdullah, M. Y., \& Ayub, S. H. (2015). Scenario on health information seeking in Malaysia: A Systematic review. Journal of Human Development and Communication, 4, 7-20.

Hashimoto, H., \& Fukuhara, S. (2004). The influence of locus of control on preferences for information and decision making. Patient Education and Counseling, 55(2), 236-240.

International Diabetes Federation (IDF), Diabetes Atlas $9^{\text {th }}$ edition (2019). Brussels: Belgium. Available at: https://www.diabetesatlas.com

International Diabetes Federation (IDF) (2021, October 23). Available at https://www.diabetesatlas.org/en/sections/demographic-and-geographic-outline.html (Accessed on 23 October 2021)

International Diabetes Federation (IDF) (2021, October 23). Available at idf.org/our-network/regionsmembers/western-pacific/members/108-malaysia.html (Accessed on 23 October 2021)

Ishikawa H. \& Kiuchi T. (2010). Health literacy and health communication. BioPsychoSocial Medicine 4:18.

Jacobs, W., Amuta, A. O., \& Jeon, K. C. (2017). "Health information seeking in the digital age: An analysis of health information seeking behaviour among US adults. Cogent Social Sciences, 3(1), $1-11$.

Johnson, J. D. (1997). Cancer-related information-seeking. Cresskill, NJ: Hampton.

Kalantzi, S., Kostagiolas, P., Kechagias, G., Niakas, D., \& Makrilakis, K. (2015). Information seeking behaviour of patients with diabetes mellitus: a cross-sectional study in an outpatient clinic of a university-affiliated hospital in Athens, Greece. BMC Research Notes, 8(1), 48. https://doi.org/10.1186/s13104-015-1005-3

Kueh, Y.C., \& Kuan, G. (2018). Psychometric Properties of Malay Version of Summary of Diabetes Self-care activities measures among the Malay adults with Type 2 Diabetes Mellitus in Kelantan, Malaysia. Austin Diabetes Res, 3(1), 1017.

Lagoe, C., \& Atkin, D. (2015). Health anxiety in the digital age: An exploration of psychological determinants of online health information seeking. Computers in Human Behaviour, 52, 484-49. https://dx.doi.org/10.1016/j.chb.2015.06.003

Lambert, S. D., \& Loiselle, C. G. (2007). Health Information-Seeking Behaviour. Qualitative Health Research, 17(8), 1006-1019. https://doi.org/10.1177/1049732307305199

Lenz, E. (1984). Information seeking: A component of client decisions and health behaviour. ANS, 6(3), 59-71.

Lintonen, T. P., Konu, A. I., \& Seedhouse, D. (2008). Information technology in health promotion. Health Education Research, 23(3), 560-566. https://doi.org/10.1093/her/cym001

Longo, D.R. (2005). Understanding health information, communication, and information-seeking of patients and consumers: A comprehensive and integrated model. Health Expectations, 8, 189-194.

Longo, D. R., Schubert, S. L., Wright, B. A., LeMaster, J., Williams, C. D., \& Clore, J. N. (2010). Health information seeking, receipt, and use in diabetes self-management. The Annals of Family Medicine, 8(4), 334-340.

Maon, S. N., Hassan, N. M., \& Seman, S. A. A. (2017). Online health information seeking behaviour pattern. Advanced Science Letters, 23(11), 10582-10585. https://doi.org/10.1166/asl.2017.10107

Mathialagan, A. G., JA, J. A., Dinesh, M., Azra, N., Selvaganapathi, G., Harikrishnan, T., \& Vikneswaran, S. (2018). Patient attitudes and health information features as predictors of health promotion in Malaysia. Journal of Advanced Pharmacy Education \& Research| Apr-Jun, 8(2). 
DOI: https://doi.org/10.47405/mjssh.v6i12.1203

Milewski, J., \& Chen, Y. (2010). Barriers of obtaining health information among diabetes patients. Studies in health technology and informatics, 160(Pt 1), 18-22.

National Diabetes Registry Report 2009-2012, Chapter 2 Malaysian Diabetes Profiling.

National Health and Morbidity Survey 2015 (NHMS 2015). Vol. II: Non-Communicable Diseases, Risk Factors \& Other Health Problems; 2015.

National Health and Morbidity Survey (NHMS) 2019 (NMRR-18-3085-44207). Non-communicable diseases, healthcare demand, and health literacy: Key Findings.

Niederdeppe, J., Hornik, R. C., Kelly, B. J., Frosch, D. L., Romantan, A., Stevens, R. S., \& Schwartz, J. S. (2007). Examining the dimensions of cancer-related information seeking and scanning behaviour. Health communication, 22(2), 153-167.

Qusaier, R.A., Al-Tqiqi, Y.E., Al-Ahmadi, H.S., Alqarni, A., \& Felemban, A.M. (2017). Information Seeking Behaviour among Diabetic Patients Attending Primary Healthcare in Western Saudi Arabia: A Cross-Sectional Two-Center Study.

Robertson, J., Akhtar, S., Petrie, J., Brown, F., Jones, G., Perry, C., \& Paterson, K. (2005). How do people with diabetes access information? Practical Diabetes International, 22(6), 207210. https://doi.org/10.1002/pdi.818

Shaw, S.J., Huebner, C., Armin, J., Orzech, K. \& Vivian, J. (2009). The role of culture in health literacy and chronic disease screening and management. J. Immigrant Minority Health 11(6): 460-467.

Shi, H.-J.,Nakamura, K., \& Takano, T. (2004). Health values and health information-seeking in relation to positive change of health practice among middle-age urban men. Preventive Medicine, 39, 1164-1171.

Vahdat, S., Hamzehgardeshi, L., Hessam, S., \& Hamzehgardeshi, Z. (2014). Patient involvement in health care decision making: a review. Iranian Red Crescent medical journal, 16(1), e12454. https://doi.org/10.5812/ircmj.12454

Wilson, T. D. (1997). Information behaviour: An interdisciplinary perspective. Information Processing \& Management, 33(4), 551-572. https://doi.org/10.1016/s0306-4573(97)00028-9

World Health Organization (2017). Shanghai declaration on promoting health in the 2030 agenda for sustainable development.

Yu, F. Q., Murugiah, M. K., Khan, A. H., \& Mehmood, T. (2015). Meta-synthesis exploring barriers to health seeking behaviour among Malaysian breast cancer patients. Asian Pac J cancer prev, $16(1), 145-152$.

Zhang, X., Foo, S., Majid, S., Chang, Y.-K., Dumaual, H. T. J., \& Suri, V. R. (2020). Self-care and health- information seeking behaviours in diabetic patients in Singapore. Journal of Health Communication, 35(8), 994-1003. https://doi.org/10.1080/10410236.2019.1606134 